Original Research Paper

\title{
Numerical Study of Natural Convection in a Square Porous Cavity Thermally Modulated on Both Side Walls
}

\author{
Giovani E.B. Malomar, Abdoulaye Gueye, Cheikh Mbow, Vieux B. Traore and Aboubaker C. Beye \\ Department of Physics, Faculty of Science and Technology, University Cheikh Anta Diop, Dakar, Senegal
}

\section{Article history}

Received: 30-06-2016

Revised: 11-08-2016

Accepted: 12-08-2016

Corresponding Author: Giovani E.B. Malomar Department of Physics, Faculty of Science and Technology, University Cheikh Anta Diop, Dakar, Senegal

Email: giovanieliemalomar@yahoo.fr

\begin{abstract}
The numerical study of natural convection in a square porous cavity saturated by a Newtonian fluid is presented in this study. The vertical walls are subjected to temperatures varying sinusoidally in time and phase opposition while the upper and lower horizontal walls are thermally adiabatic. Darcy model is used, it is also assumed the fluid studied is incompressible and obeys the Boussinesq approximation. The focus is on the effect of the modulation frequency $(10 \leq \omega \leq 100)$ and the Rayleigh number $(10 \leq \mathrm{Ra} \leq 1000)$ on the structure of the flow and transfer thermal. The results show that the extremal stream functions $\left(\psi_{\max }\right.$ and $\left.\psi_{\min }\right)$, the average Nusselt number at the hot $\left(T_{c}\right)$ and cold $\left(T_{f}\right)$ walls respectively Nucmoy and Nufmoy are periodic and periods equal to that excitatory temperatures to the range of parameters considered in this study. The results show also that oscillatory heating causes the appearance of secondary flow, whose amplification depends on the frequency of modulation of the imposed temperature. The results are shown in terms of streamlines and isotherms during a flow cycle.
\end{abstract}

Keywords: Natural Convection, Sinusoidal Temperature, Porous Media, Numerical Study

\section{Introduction}

The natural convection in confined porous media saturated by fluid is fundamental in the fields of engineering and physics. This interest arises from the importance of this heat transfer mode in various engineering fields such as storage of the thermal energy, solar energy collectors, thermal design for the buildings, cooling of electronic components (Alves and Altemani, 2012; Kuznetsov and Sheremet, 2008; Sheremet and Pop, 2014), the underground spread of pollutants (Bagchi and Kulacki, 2013).

The literature on the convective flow in porous media is abundant. An excellent review of most of these studies is in the books (Nield and Bejan, 2006; Pop and Ingham, 2001; Vafai, 2005; Ingham and Pop, 2005) that give a complete overview of the current state of research in this area.

However most of these studies, theorical and experimental, on side convection, consider boundary conditions thermal constant (temperature or flux constant heat). Excellent review articles including one written by (Baytas and Pop, 2002) give detailed accounts results obtained with these boundaries conditions. However, these boundary conditions do not reflect what is encountered in many practical situations where the temperature gradient is a function of time. This is the case of electronic components that dissipate power intermittently during operation on/of. Mastering the gradient behavior heat in these real situations can be used to control the flow convective. For example, it may be used to control the quality and structure of a solid resulting from solidification of an alloy by influencing the process of transport. The effect of the modulation of temperature on natural convection in cavity fluid medium was several time studied. For example, (Schaladow et al., 1989) studied the case of a cavity subjected to a temperature which increases linearly each time. Both considered simulations (numerical and experimental) show that the flow and temperature field are very little affected by these boundary conditions. For their part, (Kazmierczak and Chinoda, 1992) have numerically studied the effect of temperature varying sinusoidally in time on fluid flow and heat transfer in a square cavity. These authors showed that the heat transfer means in time is substantially insensitive to the periodic change of the wall temperature. Lage and Bejan (1993) studied enclosures with one sidewall heated using 
a pulsating heat flux and the other sidewall cooled at constant temperature. They showed that at high Rayleigh numbers, the buoyancy-driven flow has the tendency to resonate to the periodic heating that has been supplied from the side. Abourida et al. (1998) have examined the effect of the imposed sinusoidal temperatures and the thermophysical ones on the fluid flow and heat transfer within the cavity. They showed that all the obtained solutions concerning the fluid flow are periodic, with a period identical to that imposed to the variable temperatures. Note that many of these works consider cavities in which the temperature of the cold wall is maintained constant. However, very little work is done on natural convection in a cavity filled with a porous medium with boundary conditions thermal periodic in time and using the Darcy model. Some of the documents dealing with this problem are (Saeid, 2005).

The main objective of the present study is to contribute to the enrichment of the kind of problem by examining the effect of the imposed sinusoidal temperatures parameter (frequency) and the Rayleigh number on the structure of the flow and transfer thermal.

In this study, we study numerically natural convection unsteady in a square cavity, filled with a porous medium and the vertical walls are subjected to temperatures sinusoidally varying with time, oscillating around fixed average values and in phase opposition. The horizontal walls are thermally adiabatic and Darcy model was used.

\section{Materials and Methods}

A schematic geometry of the problem is shown in Fig. 1, where x' and y' are the cartesian coordinates and $H^{\prime}$ is the size of the walls. This is a square porous cavity two-dimensional and the horizontal walls are assumed to be thermally adiabatic. All walls of the cavity are assumed to be impermeable. At the same, the vertical walls, left (hot wall $T_{c}$ ) and right (cold wall $T_{f}$ ) are subjected to temperatures sinusoidally varying with time and in phase opposition such that:

$$
\begin{aligned}
& T_{c}^{\prime}=T_{R}+\Delta T^{\prime}+a^{\prime} \sin \left(\omega^{\prime} t^{\prime}\right) \\
& T_{f}^{\prime}=T_{R}+a^{\prime} \sin \left(\omega^{\prime} t^{\prime}+\phi^{\prime}\right)
\end{aligned}
$$

where, $T_{R}$ is the reference temperature (in our study $\left.T_{R}=\overline{T_{f}^{\prime}}\right), \quad \Delta T^{\prime}=\overline{T_{c}^{\prime}}-\overline{T_{f}^{\prime}} \quad$ is the average temperature difference, a' represents the amplitude of the modulation, $\phi^{\prime}$ the phase angle and $\omega^{\prime}$ the modulation frequency.

The Darcy-Boussinesq approximation is employed. Isotropy, homogeneity and local thermal equilibrium in the porous medium are assumed. Under these assumptions, the equations governing the problem is the equation continuity, the equation of motion and the energy equation, respectively (Nield and Bejan, 2006):

$$
\begin{aligned}
& \frac{\partial u^{\prime}}{\partial x^{\prime}}+\frac{\partial v^{\prime}}{\partial y^{\prime}}=0 \\
& \frac{\partial v^{\prime}}{\partial x^{\prime}}-\frac{\partial u^{\prime}}{\partial y^{\prime}}=\frac{k g \rho_{0} \beta_{T}}{\mu} \frac{\partial T^{\prime}}{\partial x^{\prime}} \\
& \sigma \frac{\partial T^{\prime}}{\partial t^{\prime}}+u^{\prime} \frac{\partial T^{\prime}}{\partial x^{\prime}}+v^{\prime} \frac{\partial T^{\prime}}{\partial y^{\prime}}=\alpha \nabla^{2} T^{\prime}
\end{aligned}
$$

where, $u^{\prime}$ and $v^{\prime}$ are the velocity components along $x^{\prime}$. and $y^{\prime}, \mathrm{T}^{\prime}$ is the fluid temperature, $t^{\prime}$ is the time, $\beta_{T}=$ $1 / \rho_{0}\left(\partial \rho / \partial T^{\prime}\right)_{p}$ is the coefficient of thermal expansion. The quantities $\sigma$ and $\alpha$ are defined by $\alpha=k_{m} /(\rho c)_{F}$ and $\sigma=$ $(\rho c)_{m} /(\rho c)_{F}$ where $k_{m}$ is the thermal conductivity (solid phase + fluid phase).

Equations 3-5 are subject to the following boundary and initial conditions:

$$
\begin{aligned}
& u^{\prime}\left(x^{\prime}, y^{\prime}, 0\right)=v^{\prime}\left(x^{\prime}, y^{\prime}, 0\right)=0 ; T^{\prime}\left(x^{\prime}, y^{\prime}, 0\right)=0 \\
& u^{\prime}\left(0, y^{\prime}, t^{\prime}\right)=0 ; T^{\prime}\left(0, y^{\prime}, t^{\prime}\right)=T_{c}^{\prime} \\
& u^{\prime}\left(H^{\prime}, y^{\prime}, t^{\prime}\right)=0 ; T^{\prime}\left(H^{\prime}, y^{\prime}, t^{\prime}\right)=T_{f}^{\prime} \\
& v^{\prime}\left(x^{\prime}, 0, t^{\prime}\right)=0 ; \partial T^{\prime}\left(x^{\prime}, 0, t^{\prime}\right) / \partial y^{\prime}=0 \\
& v^{\prime}\left(x^{\prime}, H^{\prime}, t^{\prime}\right)=0 ; \partial T^{\prime}\left(x^{\prime}, H^{\prime}, t^{\prime}\right) / \partial y^{\prime}=0
\end{aligned}
$$

One can introduce a stream function $\psi^{\prime}$ defined by $u$ ' $=\partial \psi^{\prime} / \partial y^{\prime}$ and $v^{\prime}=-\partial \psi^{\prime} / \partial x^{\prime}$ so that Equation 3 is satisfied. Then the governing Equations 3-5 can be rewritten with the following dimensionless variables:

$$
\begin{aligned}
& (x, y)=\left(\frac{x^{\prime}}{H^{\prime}}, \frac{y^{\prime}}{H^{\prime}}\right) ; t=\frac{t^{\prime} \alpha}{\sigma H^{\prime 2}} ; \tau=\frac{\tau^{\prime} \alpha}{\sigma H^{\prime 2}} \\
& T=\frac{T^{\prime}-\overline{T_{f}^{\prime}}}{\overline{T_{c}^{\prime}}-\overline{T_{f}^{\prime}}} ; \psi=\frac{\psi^{\prime}}{\alpha} ; a=\frac{a^{\prime}}{\overline{T_{c}^{\prime}}-\overline{T_{f}^{\prime}}}
\end{aligned}
$$

$\dot{\tau}$, represents the dimensional period and is connected to the modulation, frequency dimensional by the following equation $\omega^{\prime}=2 \pi / \tau^{\prime}$.

Substituting Equation 7 in Equations 3-5 we obtain the following dimensionless governing equations:

$$
\begin{aligned}
& \frac{\partial^{2} \psi}{\partial x^{2}}+\frac{\partial^{2} \psi}{\partial y^{2}}=-R a \frac{\partial T}{\partial x} \\
& \frac{\partial T}{\partial t}+\frac{\partial \psi}{\partial y} \frac{\partial T}{\partial x}-\frac{\partial \psi}{\partial x} \frac{\partial T}{\partial y}=\frac{\partial^{2} T}{\partial x^{2}}+\frac{\partial^{2} T}{\partial y^{2}}
\end{aligned}
$$




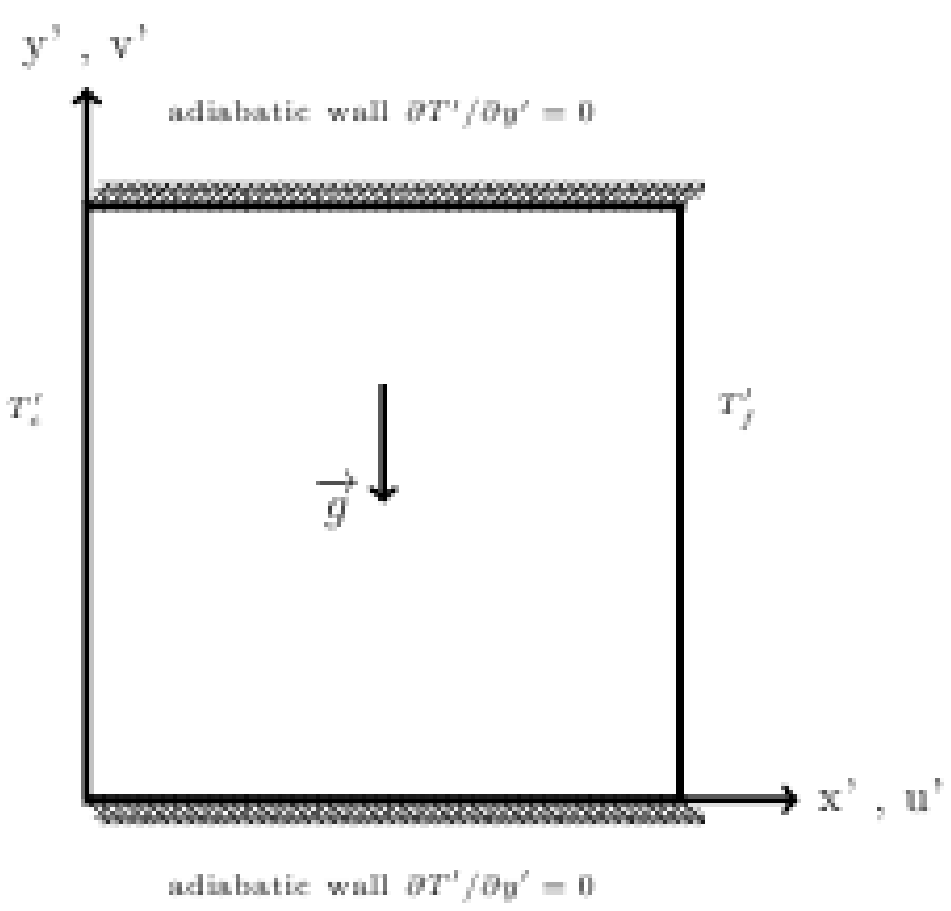

Fig. 1. Schematic diagram of the physical model and coordinate system

Table 1. Comparison of the average Nusselt number of the hot wall

\begin{tabular}{llll}
\hline & Nucmoy & \\
& - & & \\
Authors & $\mathrm{Ra}=10$ & $\mathrm{Ra}=100$ & $\mathrm{Ra}=1000$ \\
\hline Walter and Homsy (1978) & - & 3.097 & 12.96 \\
Bejan (1979) & - & 4.2 & 15.8 \\
Beckermann et al. (1986) & - & 3.113 & - \\
Moya and Ramos (1987) & 1.065 & 2.801 & - \\
Manole and Lage (1992) & - & 3.118 & 13.637 \\
Baytas and Pop (2002) & 1.079 & 3.16 & 14.06 \\
Present results & 1.078 & 3.12 & 15.6 \\
\hline
\end{tabular}

Associated with initial and boundary conditions:

$$
\begin{aligned}
& \psi(x, y, 0)=0 ; T(x, y, 0)=0 \\
& \psi(0, y, t)=0 ; T(0, y, t)=T_{c} \\
& \psi(1, y, t)=0 ; T(1, y, t)=T_{f} \\
& \psi(x, 0, t)=0 ; \partial T(x, 0, t) / \partial y=0 \\
& \psi(x, 1, t)=0 ; \partial T(x, 1, t) / \partial y=0
\end{aligned}
$$

Here $T_{c}=1+a \sin (\omega t) ; T_{f}=a \sin (\omega t+\phi)$ and $R a=$ $k \rho_{0} g \beta_{T} \Delta T^{\prime} H^{\prime} / \mu \alpha$ represents the Rayleigh number. The physical quantities of interest are the average Nusselt numbers at the vertical walls. The numbers of local Nusselt are defined as:

$$
N u c=-\left(\frac{\partial T}{\partial x}\right)_{x=0} ; N u f=-\left(\frac{\partial T}{\partial x}\right)_{x=1}
$$

At each time $t$ the average Nusselt numbers at the vertical walls (hot and cold) are defined by, respectively:

$$
\begin{aligned}
& \text { Nucmoy }=-\int_{0}^{1}\left(\frac{\partial T}{\partial x}\right)_{x=0} d y \\
& \text { Nufmoy }=-\int_{0}^{1}\left(\frac{\partial T}{\partial x}\right)_{x=1} d y
\end{aligned}
$$

The equations of motion (8) and energy (9) associated with the boundary conditions (10a-10e) are discretized by a finite difference scheme, centred and accurate to the second order. The energy equation is then solved by the implicit method of Alternating Directions (ADI). The linear discretized equations were solved by Thomas algorithm. For equation of motion, the obtained linear discretized equation was solved by the successive over-relaxation method. Uniform grids have been selected in both the $\mathrm{x}$ and $\mathrm{y}$ direction.

We have developed a numerical code with Fortran 95. The calculation stops when between two time steps, the following conditions are satisfied simultaneously by the stream function and the temperature:

$\max \left(\left|\frac{\psi^{n+1}-\psi^{n}}{\psi^{n}}\right|\right) \leq 10^{-8}$ 
$\max \left(\left|\frac{T^{n+1}-T^{n}}{T^{n}}\right|\right) \leq 10^{-8}$

Preliminary tests on the influence of the mesh have allowed us to retain a uniform mesh size of $120 * 120$. The time step used is $10^{-4}$. The present numerical code have been validated against the works of (Walker and Homsy, 1978; Bejan, 1979; Beckerman et al., 1986; Moya and Ramos, 1987; Manole and Lage, 1992; Baytas and Pop, 2002) for the steady state natural convection in a square porous cavity with isothermal vertical and adiabatic walls. Table 1 shows the values of the average Nusselt number computed for various Rayleigh numbers in the range 10-1000 in comparison with other authors.

Note that all numerical simulations are initialized by considering a conductive state and constant heating conditions. When steady regime is established, we introduce the excitatory temperatures and expecte the establishment of a periodic regime.

\section{Results and Discussion}

In this section, we present the effect of the modulation frequency on temporal evolution of the extremal stream functions $\left(\psi_{\max }, \psi_{\min }\right)$ and of the average Nusselt numbers (Nucmoy, Nufmoy). The results are presented in terms of flow structure (stream lines) and heat transfer (isotherms).

\section{Results Relating to Constant Heating}

Before discussing the results for the oscillatory regime, we produced streamlines and isotherms in steady state $(\mathrm{a}=0)$ for different values of the Rayleigh number $\mathrm{Ra}$ $=(10-100-1000)$. Note that in the case of constant heating $(\mathrm{a}=0)$, the flow in the cavity remains monocell, consisting of a positive cell rotating in clockwise and having a symmetry relative to the center of the cavity (Fig. 2).
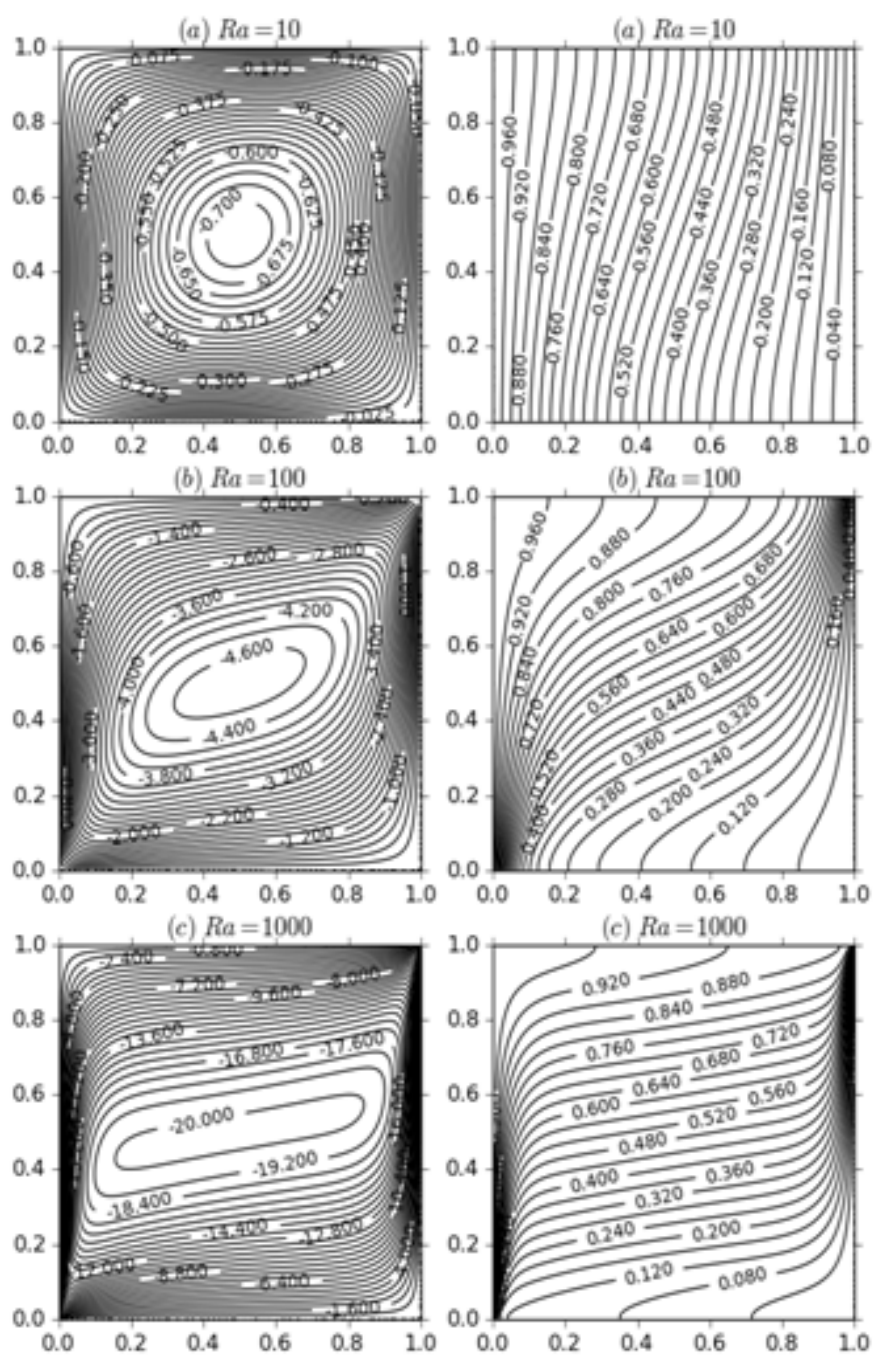

Fig. 2. Streamlines (left) and isotherms (right) for $\mathrm{Ra}=10 ; \mathrm{Ra}=100 ; \mathrm{Ra}=1000$ : Steady state solution 

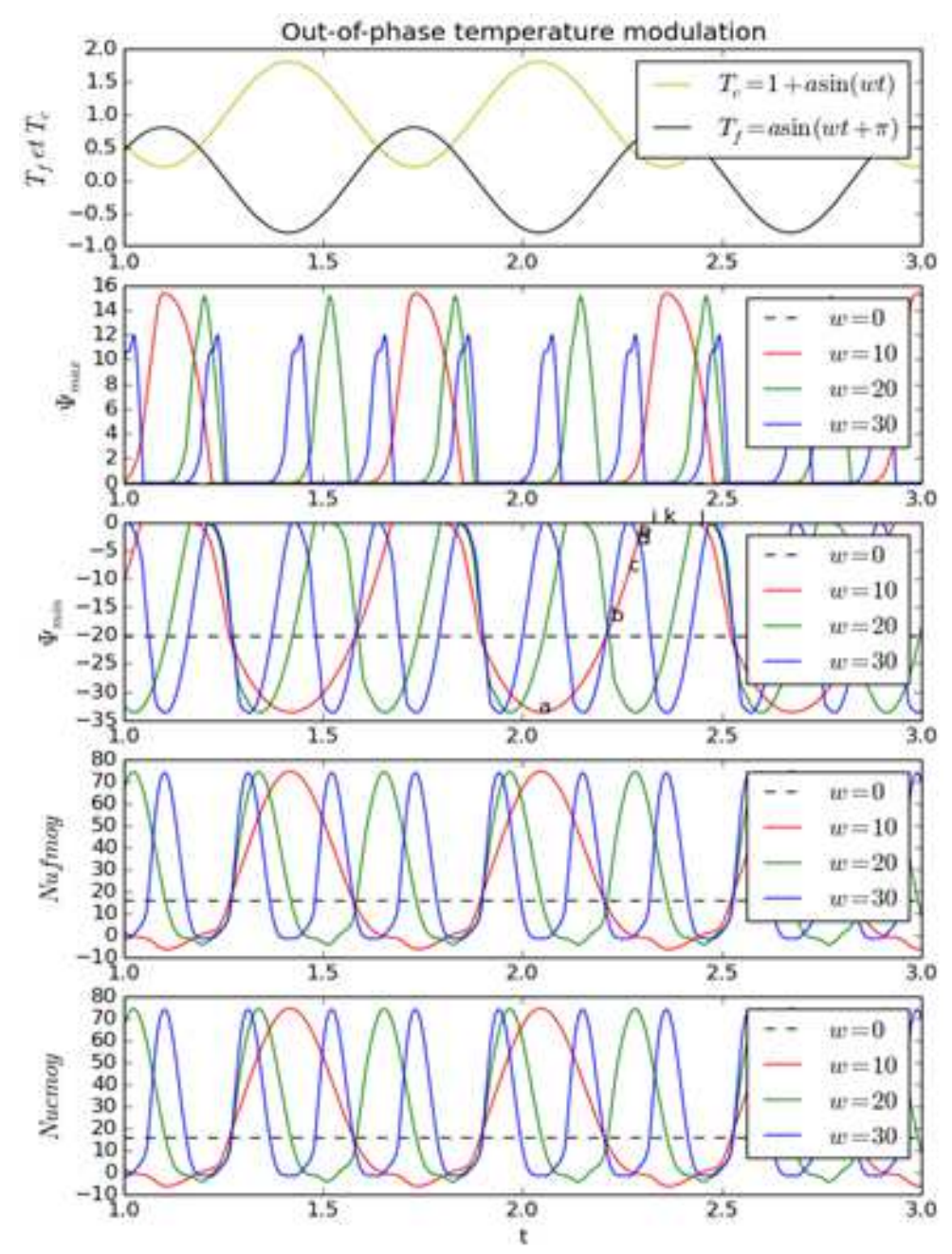

Fig. 3. Effect of $\omega$ for $\mathrm{a}=0.8$ and $\mathrm{Ra}=1000$ on (from top to bottom): (a) $\mathrm{Tc}(\mathrm{t})$ and $\mathrm{Tf}(\mathrm{t}),(\mathrm{b}) \psi \max (\mathrm{t}),(\mathrm{c}) \psi \min (\mathrm{t}),(\mathrm{d}) \mathrm{Nufmoy}(\mathrm{t})$ and (e) Nucmoy(t)

However, its intensity remains low to moderate values of $\mathrm{Ra}(\mathrm{Ra}=10$ and $\mathrm{Ra}=100)$ and becomes significant with the increase in $\mathrm{Ra}(\mathrm{Ra}=1000)$. For $\mathrm{Ra}=1000$, structure isotherms is almost stratified in the central region of cavity and strong thermal gradients are noted in the areas surrounding the active walls.

\section{Results Relating to Variable Heating}

\section{Influence of the Modulation Frequency}

To highlight the effect of $\omega$ frequency modulation, we present the temporal evolution of the functions $\psi_{\max }$, $\psi_{\min }$, Nucmoy and Nufmoy for heating oscillatory under the following conditions $\mathrm{a}=0.8 ; \mathrm{Ra}=10^{3}$ and $10 \leq \omega \leq$ 30. Figures $3 \mathrm{a}-3 \mathrm{e}$ show respectively the evolution temporal functions, $T_{c}$ and $T_{f}, \psi_{\max }, \psi_{\min }$, Nucmoy and Nufmoy in the case where the temperatures excitatory evolve in phase opposition. The curves of Fig. 3b-3e show that all the solutions obtained are periodic, periods equal to those the imposed excitatory temperatures. In range $10 \leq \omega \leq 30$, the amplitudes of the functions presented remain very close except for $\psi_{\max }(t)$ where there is a notable decrease in the amplitude when $\omega$ go 20 to 30 . For this heating mode is also observed better competition between positive and negative cells, resulting in a cancellation of $\psi_{\min }$ during part of the cycle. During this time interval, the flow is monocellular, characterized by a presence of a positive cell turning clockwise and of which intensity decreases when $\psi_{\min }$ tends to 0 .

Figures $3 \mathrm{~d}-3 \mathrm{e}$ show that during part of the cycle, the average Nusselt numbers Nucmoy and Nufmoy take negative values. These negative values found, are justified by the fact that for amplitudes $(a>0.5)$ the cold wall acquires temperatures higher than the wall supposed to be hot. This then results in a transfer of heat from the cold wall inwardly of the cavity as soon as this wall becomes hotter than the surrounding fluid. Thereafter the fluid surrounding the hot wall becomes hotter than this wall it results a heat transfer from the fluid outwardly the cavity via the wall hot. 

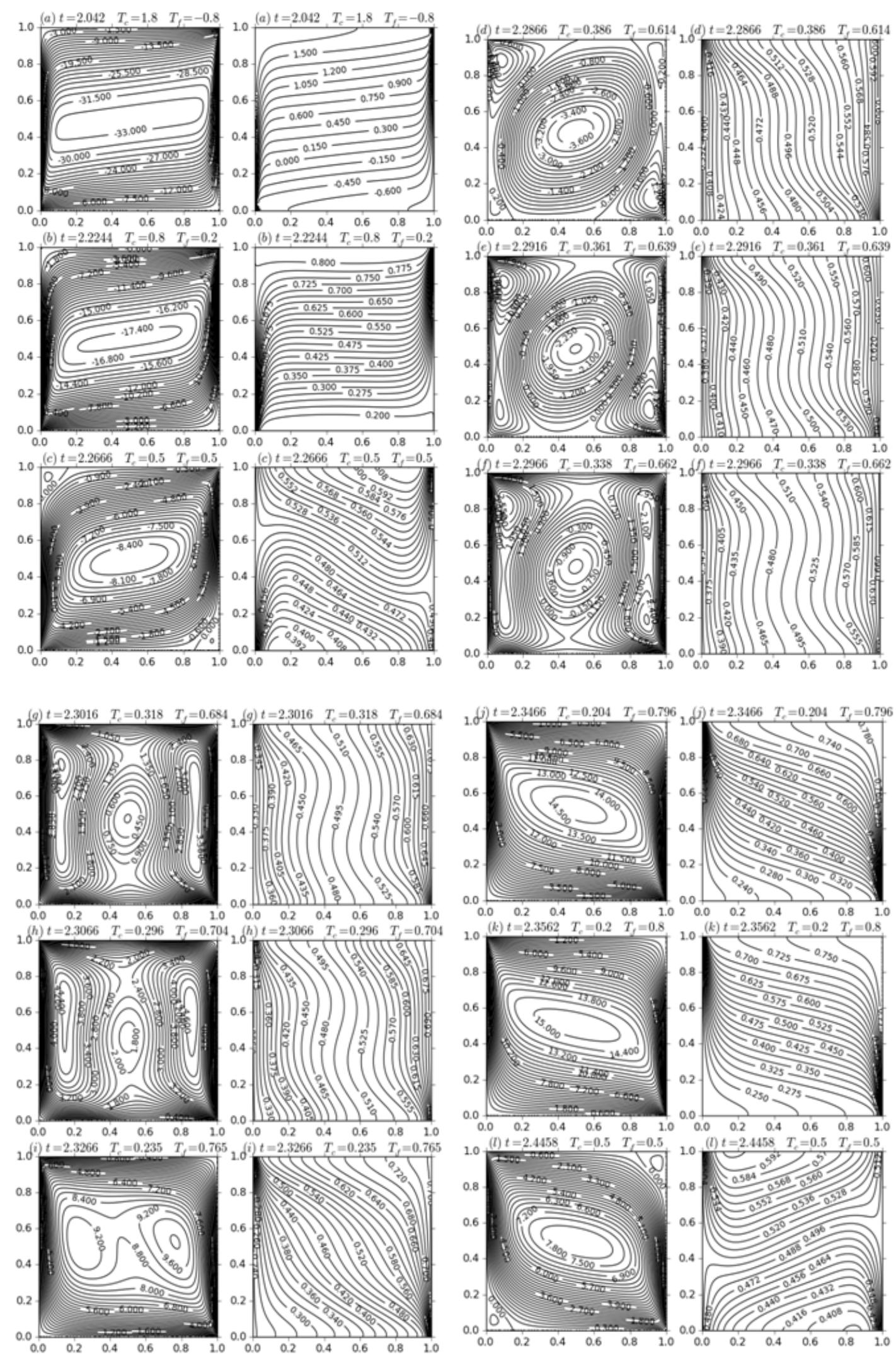

Fig. 4. (a)-(1): Streamlines (left) and isotherms (right) over half cycle for $\mathrm{Ra}=1000, \mathrm{a}=0.8$ and $\omega=10$ 
However, we note that the most important effects of frequency modulation $\omega$ are rated for high values of this parameter $(\omega \geq 100)$. But for convenience we chose to study the functions for $10 \leq \omega \leq 30$ because even for this range frequency, important differences qualitative and quantitative are observed.

\section{Streamlines and Isotherms}

To understand details of flow and heat transfer caused by applying the boundary conditions oscillatory thermal, we have produced the stream lines and isotherms during a cycle for $\mathrm{Ra}=10^{3}, \mathrm{a}=0.8$ and $\omega=$ 10. Figures $4 \mathrm{a}-41$ present stream lines (left) and isotherms (right) at times corresponding to the letters a, b, c, d, ...1, (Fig. 3). Note that these moments are defined in the order alphabet and some of the points (f, g, h, j) are not on the Fig. 3. They have been omitted for clarity. We note that at the moment (a), the flow regime is monocell, constituted by positive cell called main cell, rotating in clockwise direction and occupying the entire cavity. We observe also at time (a) the structure of isotherms shows stratification of the temperature around the center of the cavity and of strong gradients thermal around the active walls (Fig. 4a). Evolving in the cycle there is a weakening of the main cell (Fig. 4b). This weakening of the main cell is further complicated by the occurrence of two negative recirculation cells, one at the upper left corner and the other at the lower right corner (Fig. 4c). The appearance of these two negative cells cause spacing of the isotherms in the vicinity of active walls whence a heat transfer reduction. At time (d), in addition of two initial negative cells that are amplified, appears two other negative cells at the two remaining corners of the cavity, resulting in a significant weakening of the main cell whence heat transfer reduction illustrated by the isotherms structure (Fig. 4d). Thereafter, we note that the negative cells on the same side (left and right) merge and lead to the total destruction of the main cell positive (Fig. 4e and 4f) in favour a main negative cell rotating in counter-clockwise, which gradually establishes (Fig. $4 \mathrm{~g}, 4 \mathrm{~h}$ and $4 \mathrm{i}$ ) and ends up occupying the entire cavity (Fig. $4 \mathrm{j}$ and $4 \mathrm{k}$ ). Between times $\mathrm{e}, \mathrm{f}, \mathrm{g}, \mathrm{h}$ and $\mathrm{i}$ the isotherms structure attests that heat transfer are virtually reduced to zero, unlike the moments $\mathrm{j}$ and $\mathrm{k}$ where the distribution of isotherms characterizing the monocell flow mode appears again.

In the second part of the cycle, the positive cells will play a role similar to the one played by the negative cell during the first part of the cycle. However the first two positive recirculation cells appear in the corner of level upper right and lower left, then appear the two other cells positive recirculation at the two remaining corners. These positive cells will unite in pairs to the detriment of the main negative cell, that ends up by disappears to leave any room for the new dominant positive cell.

\section{Conclusion}

The numerical study of unsteady natural convection in a porous cavity square whose side walls are subjected to temperatures sinusoidal and in opposite phase was investigated. The mathematical model used is that of Darcy in the Boussinesq approximation. The algorithm was validated by direct comparison with previously published work and the results were considered in good agreement. Stream lines and isotherms were produced for $\mathrm{Ra}=10^{3}, \mathrm{a}=$ 0.8 and $\omega=10$ in the case of variable heating and $\mathrm{Ra}=(10$ $10^{2}-10^{3}$ ) for the case of constant heating. Based on the results found in this study, we conclude that:

- Oscillatory heating causes the appearance of secondary flow, whose amplification depends on the frequency of modulation of the imposed temperature

- The imposed oscillatory heating improves thermal transfer compared to the constant heating and constitutes the best way to remove the heat to the outside environment

- When both temperatures evolve in opposite phase, important differences are noted in terms of flow structure and of transfers heat unlike the case of constant heating; depending on the purpose, these differences can be exploited by the modeler

This configuration is a model for cooling components generating heat in electronic plates.

\section{Funding Information}

The authors have no support or funding to report.

\section{Author's Contributions}

Giovani Elie Baptista Malomar: Conducted the numerical work and wrote the initial draft of the paper.

Abdoulaye Gueye: Contributed in data analysis and revising the manuscript.

Cheikh Mbow: Contributed in substantial revision and improvement of the first draft of the manuscript.

Vieux Boukhaly Traore: Contributed in data analysis and revising the manuscript.

Aboubaker Chedikh Beye: Contributed in substantial revision and improvement of the first draft of the manuscript.

\section{Ethics}

The authors have read and approved the manuscript and no ethical issues involved.

\section{References}

Abourida, B., M. Hasnaoui and S. Douamna, 1998. Natural convection in a square cavity with vertical boundaries submitted to periodic temperatures. Rev. Gen. Therm., 37: 788-800. DOI: 10.1016/S0035-3159(98)80005-6 
Alves, T.A. and C.A.C. Altemani, 2012. An invariant descriptor for heaters temperature prediction in conjugate cooling. Int. J. Therm. Sci., 58: 92-101. DOI: 10.1016/j.ijthermalsci.2012.03.007

Bagchi, A. and F.A. Kulacki, 2013. Natural Convection in Superposed Fluid-Porous Layers. 1st Edn., Springer, New York, ISBN-10: 1461465761, pp: 80.

Baytas, A.C. and I. Pop, 2002. Free convection in a square porous cavity using a thermal nonequilibrium model. Int. J. Thermal Sci., 41: 861-870. DOI: $10.1016 / \mathrm{S} 1290-0729(02) 01379-0$

Beckerman, C., R. Viskanta and S. Ramadhyani, 1986. A numerical study of non-darcian natural convection in a vertical enclosure filled with a porous medium. Numer. Heat Transf., 10: 557-570. DOI: $10.1080 / 10407788608913535$

Bejan, A., 1979. On the boundary layer regime in a vertical enclosure filled with a porous medium. Lett. Heat Mass Transfer, 6: 93-102. DOI: 10.1016/0094-4548(79)90001-8

Ingham, D.B. and I. Pop, 2005. Transport Phenomena in Porous Media III. 1st Edn., Elsevier, Oxford, ISBN-10: 0080543189, pp: 450.

Kazmierczak, M. and Z. Chinoda, 1992. Buoyancydriven flow in an enclosure with time periodic boundary conditions. Int. J. Heat Mass Transfer, 35: 1507-1518. DOI: 10.1016/0017-9310(92)90040-Y

Kuznetsov, G.V. and M.A. Sheremet, 2008. New approach to the mathematical modeling of thermal regimes for electronic equipment. Russ. Microelectron., 37: 131-138. DOI: $10.1134 / \mathrm{S} 1063739708020078$

Lage, J.L. and A. Bejan, 1993. The resonance of natural convection in an enclosure heated periodically from the side. Int. J. Heat Mass Transfer, 36: 2027-2038. DOI: $10.1016 / \mathrm{S} 0017-9310(05) 80134-6$

Manole, D.M. and J.L. Lage, 1992. Numerical benchmark results for natural convection in a porous medium cavity. Heat Mass Transfer Porous Media, 216: 55-60.

Moya, S.L. and E. Ramos, 1987. Numerical study of natural convection in a tilted rectangular porous material. Int. J. Hem Mass Transfer, 30: 741-756. DOI: 10.1016/0017-9310(87)90204-3

Nield, D.A. and A. Bejan, 2006. Convection in Porous Media. 3rd Edn., Springer Science and Business Media, New York, ISBN-10: 0387290966, pp: 640.

Pop, I. and D.B. Ingham, 2001. Convective Heat Transfer: Mathematical and Computational Modelling of Viscous Fluids and Porous Media. 1st Edn., Elsevier, Amsterdam, ISBN-10: 0080530001, pp: 668 .

Saeid, N.H., 2005. Natural convection in a square porous cavity with an oscillating wall temperature. Arabian J. Sci. Eng., 31: 35-46.
Schaladow, S.G., J.C. Patterson and R.L. Street, 1989. Transient flow in a side-heated cavity at high Rayleigh number: A numerical study. J. Fluid Mech., 200: 121-148. DOI: 10.1017/S0022112089000595

Sheremet, M.A. and I. Pop, 2014. Natural convection in a square porous cavity with sinusoidal temperature distributions on both side walls filled with a Nanofluid: Buongiorno's mathematical model. Transp. Porous Med., 105: 411-429. DOI: $10.1007 / \mathrm{s} 11242-014-0375-7$

Vafai, K., 2005. Handbook of Porous Media. 2nd Edn., CRC Press, New York, ISBN-10: 0415876389, pp: 784.

Walker, K.L. and G.M. Homsy, 1978. Convection in a porous cavity. J. Fluid Mechan., 87: 449-474. DOI: $10.1017 / \mathrm{S} 0022112078001718$

\section{Nomenclature}

a Dimensionless amplitude

g Gravity intensity, m.s s $^{-2}$

$\mathrm{k}$ Permeability of the porous medium, $\mathrm{m}^{2}$

$\mathrm{t} \quad$ Dimensionless time

$\mathrm{T} \quad$ Dimensionless temperature

$(\mathrm{x}, \mathrm{y}) \quad$ Dimensionless coordinates

$\mathrm{Ra} \quad$ Rayleigh number for porous medium

$\Delta \mathrm{T}$, Average temperature difference, $\mathrm{K}$

Nucmoy Average Nusselt number at the hot wall

Nufmoy Average Nusselt number at the cold wall

\section{Greek Symbols}

$\alpha \quad$ Effective thermal diffusivity, $\mathrm{m}^{2} \cdot \mathrm{s}^{-1}$

$\beta_{\mathrm{T}} \quad$ Coefficient of thermal expansion, $\mathrm{K}^{-1}$

$\mu \quad$ Dynamic viscosity, $\mathrm{kg} \cdot \mathrm{m}^{-1} \cdot \mathrm{s}^{-1}$

$\Phi$ Dimensionless phase angle

$\sigma \quad$ Ratio of heat capacity of porous medium to that of fluid

$\omega$ Dimensionless frequency

$\tau \quad$ Dimensionless period

$\psi \quad$ Dimensionless stream function

$\rho_{\mathrm{o}} \quad$ Reference density of fluid, kg. $\mathrm{m}^{-3}$

\section{Superscript}

(') Dimensional variables

(-) Average values

\section{Subscript}

c Hot

f Cold

F Fluid

min Minimum value

max Maximum value 